\title{
TORSION OF OTHERWISE HEALTHY OVARY HAS A WORSE PROGNOSIS THAN TORSION OF PATHOLOGIC OVARY IN CHILDREN
}

Ollivier Margot, MD ${ }^{1}$; Sfar Mohamed Sami, MD ${ }^{1}$; Teissier Benoit, MD ${ }^{1}$; Ben Ahmed Yosra, $\mathrm{MD}^{1}$; Coffy Amandine, $\mathrm{MD}^{2}$; Garnier Sarah, $\mathrm{MD}^{1}$; Guibal Marie Pierre, MD ${ }^{1}$; Sultan Charles, $\mathrm{MD} \mathrm{PhD}{ }^{3,4}$; Paris Françoise, $\mathrm{MD} \mathrm{PhD}^{3,4}$; Kalfa Nicolas, MD PhD ${ }^{1,4}$.

1-Department of Pediatric Surgery and Urology, Lapeyronie Hospital, University Hospital of Montpellier and University of Montpellier, Montpellier, France

2-University Institute of Clinical Research, University of Montpellier, France

3-Department of Pediatric Endocrinology, Arnaud de Villeneuve Hospital, University Hospital of Montpellier, Montpellier, France.

4- Rare Disease National Reference Center (Constitutive South) DEVGEN.

Corresponding Author: Kalfa Nicolas, Departement of Pediatric Surgery and Urology, Lapeyronie Hospital, University Hospital of Montpellier-371 avenue du Doyen Gaston Giraud-34295 MONTPELLIER cedex 5. Tel : 0033-467338784. Email : nicolaskalfa@gmail.com.

No Conflicts of Interest for all authors. 
INTRODUCTION

2

Ovarian torsion is defined as a partial or total twist of the ovary around its own pedicle inducing an acute arterial ischemia. Even if its incidence is quite limited among all children and adolescent $(0.02 \%)(1,2)$, ovarian torsion remains a frequent gynecological emergency (3). Fifteen per cent of all ovarian torsions occur in children and adolescent girls (4). Pediatric cases are reported in both early infancy and around the time of puberty (5). Whatever the age of occurrence, an early recognition of the diagnosis is the best way to prevent functional consequences on the ovary. Unfortunately the clinician has to face several difficulties. Clinical symptoms of ovarian torsion remain non-specific (6), the differential diagnosis of abdominal pain in children is wide, a radiologic testing with a high specificity and sensitivity remains to be identified (7), and unnecessary surgery is still frequent (5).

Hypotrophy and atrophy are a frequent complication of an ovarian torsion. Even if a conservation of the twisted ovary is now generally admitted (1,8-10), a limited number of ovaries will be found follicular (9). Determining the prognostic factors is thus of importance and they could help to focus on the at-risk patients and to ensure an expeditive surgical treatment. To date, only one prognostic factor has been identified: the time interval between the onset of pain and the surgical management which is associated with the degree of ischemia (1). The exact duration that leads to an ovarian loss is unfortunately not precisely determined and may also depends on the number of twists and residual blood flow. Further prognostic factors are thus needed since focusing on patients with persistent and unexplained symptoms is not sufficient to reduce the morbidity of ovarian torsion.

In order to determine other prognostic factors or groups of patients at-risk of severe ovarian atrophy, this study was designed to test if any clinical symptoms, blood analysis or underlying process of the twisted ovary may influence the ovarian outcome after a torsion in pediatric patients.

\section{MATERIALS AND METHODS}

\section{Patients}

A monocentric retrospective study was performed in our pediatric surgery department from 1997 to 2016. All patients with an ovarian torsion confirmed at surgery occurring between birth and 17 years were included. Patients with prenatal ovarian torsion and isolated oviduct torsion were excluded. Collected data included the age of occurrence, clinical 
symptoms, the initially suspected diagnosis, biological data, ultrasonographic data, the time interval between the first symptoms and the surgical untwisting of the ovary and the presence or absence of an underlying ovarian disease (otherwise healthy ovary versus ovarian benign cyst or tumor). The confirmation of the diagnosis, the evaluation of the viability and the global aspect of the ovary were assessed during laparoscopy. In case of an otherwise healthy ovary, the treatment consisted in the reduction of the torsion. In case of an ovarian mass, an initial untwisting was performed. A secondary cystectomy with conservation of the adjacent parenchyma was performed if the plasma levels of alpha-fetoprotein and hCG were normal (11). In case of no detectable residual parenchyma adjacent of a voluminous mass, an ovariectomy was preferred. All patients were followed at 1 month, 6 months, 1 year and 3 years with a clinical exam and an ultrasonography. The primary outcome was the trophicity of the ovary at the end of the follow-up. This outcome was classified in 2 groups as normotrophic ovary vs atrophic (non-visible) or hypotrophic ovary (ovarian volume loss of $25 \%$ or more when compared to contralateral side). Our local ethic committee (Centre de Protection des Personnes Sud Méditerranée 4, CPPSMIV) approved this retrospective and anonymized on charts study (Ref number CPP:Q-20170901).

\section{Statistical Analysis:}

Patients' characteristics were presented using median and range for continuous variables and frequencies for categorical variables. After a descriptive analysis of the whole series, 2 groups were defined (torsion of healthy ovary vs torsion of pathologic ovaries i.e. with an ovarian mass). The comparison between these groups was assessed using Chi2 tests. Logistic regression analysis was used to evaluate the relation between the timing before surgery, the underlying ovarian disease and the final outcome. Statistical analysis was performed using SAS software 9.3 (SAS Institute, Cary, N.C.). For all tests a significance level (alpha) of 0.05 was used.

\section{RESULTS}

\section{1- Descriptive analysis}

Fifty-seven girls were included in the study. Three were lost at follow-up and 54 cases were studied. The patients 'mean age was 10 years ranging from 2 months to 17 years with a bimodal repartition as presented in figure 1 . Overall missing data was $1.46 \%$. Clinical 
symptoms were not specific (table 1). About half of patients experienced nausea $(46.3 \%$, $n=25)$, however the classical vomiting was present in only $25.9 \%$ of cases $(n=14)$. Leukocytosis above $10 \times 10^{3} / \mathrm{mm}^{3}(48.1 \%, \mathrm{n}=26)$ and C-reactive protein above $10 \mathrm{mg} / \mathrm{L}$ $(18.5 \%, \mathrm{n}=10)$ were inconstant. The time between the onset of pain and the surgery was highly variable (Figure 2) ranging from 3 hours to 10 days, median $=48 \mathrm{~h}$. The ultrasonography exhibited in all cases an ovary of increased size. An additional mass adjacent to the ovary corresponding to the spiral twist was reported in $42.6 \%$ of patients $(n=23 / 54)$. An underlying ovarian cyst or mass was suspected in 27 cases. In the 27 others, a global and homogeneous dilation of the ovary with peripheral follicles was in favor of a torsion of a healthy ovary. At the end of follow-up, $46.3 \%$ of patients had a normothrophic ovary $(n=25)$, $42.6 \%$ had either an ovarian atrophy (non-detectable ovary at US, $\mathrm{n}=14$ ) or an ovarian hypotrophy ( $\mathrm{n}=11$, mean volume loss $74 \%$, from 28 to $95 \%)$. Four patients had an ovariectomy since no residual peripheral parenchyma was identifiable around a large mass.

\section{2- Comparison between torsion of healthy vs pathologic ovaries}

Twenty-seven patients had an ovarian torsion with an underlying ovarian mass (TUOM) (cystic teratoma $n=11$, follicular cyst $n=14$, serous cystadenoma $n=2$ ). The 27 other girls had torsion of an otherwise normal and healthy ovary (THO). No malignant tumor was found in this series. It was not possible to differentiate TUOM and THO based on the clinical symptoms (Table 1). Figure 1 compares the repartition of the 2 groups according to age. Mean age of occurrence was significantly lower in THO than in TUOM ( $<<0.001$, table 1$)$. Diagnosis of an ovarian torsion was less frequently suspected on the first clinical exam in case of THO (table 1). Recurrent torsion was infrequent $(3.7 \%, n=1 / 27)$ in both groups.

\section{3- Prognostic factors}

We then compared patients with subsequent hypotrophy or atrophy of the ovary at follow up to those with normotrophic ovaries. The univariate and multivariate analyses did not identify age, clinical symptoms or biological markers as significant prognostic factors, except fever that was more frequently associated with secondary hypotrophy or atrophy ( $88.8 \%$ vs $63.7 \%, p=0.02$ ). Our study confirmed that the time period between the first symptoms and surgery is of importance. A late surgery (> 48h after the first symptoms) is more frequently associated with altered trophicity of the ovary $(n=5,46.4 \%$ vs $n=13,18.5 \%$, $\mathrm{p}=0,04)$. 
Beside this well-known effect of late management, we found that the underlying

103 disease of the ovary is a significant prognostic factor. Torsion of a healthy ovary is more frequently associated with an ovarian hypo/atrophy than TUOM $(n=20,74.1 \%$ vs $n=5,18.5 \%$ $\mathrm{p}<0.01)$. The logistic regression analysis confirmed this finding $(\mathrm{OR}=5.08, \mathrm{p}=0.01)$. We thus tried to find out which difference between THO and TUOM may explain this result. The delay before surgery tended to be shorter in TUOM than in THO but this did not reach significance (Table 1, Figure1). TUOM occurred in older girls than THO (6.7 vs 11.3 years, $\mathrm{p}<0.001)$, and more frequently after puberty $(>12$ years, $59.2 \%$ vs $11.1 \%, \mathrm{p}<0.001$, Fischer's Exact test, Figure 2).

\section{DISCUSSION}

114 Torsion of the ovary is an infrequent but classical cause of abdominal pain in young and

115 adolescent girls. The most frequent clinical symptoms (pain, nausea and vomiting) are not 116 specific $(6,12)$ and the differential diagnosis for acute abdominal pain remains wide and 117 tricky. The lack of consistent localization of pain and the low specificity of leukocytosis or 118 level of C Reactive Protein in our series may not help the clinician. A recent meta-analysis 119 based on 663 torsions confirms that the diagnosis of ovarian torsion remains a challenge with 120 a median delay of 100 hours (13). The need of an efficient and early diagnosis remains

121 mandatory since the delay before surgery is a major prognostic factor for the subsequent 122 trophicity of the ovary. Ultrasonography (US) is widely indicated for unexplained abdominal 123 pain in pediatric patients and may be quite useful here. An increased size of the ovary 124 associated with an adjacent mass corresponding to the spiral twist of the fallopian tube should raise suspicion on the diagnosis. But pitfalls for the diagnosis by US remain numerous and may include the low sensitivity of absent arterial blood flow (residual Doppler signals), confusion with hemorrhagic cysts and difficult identification of small ovaries in young children that are referred early. The overall sensitivity of US remains limited in the literature (79\%) (13), this exam does not prevent from frequent ovarian hypotrophy or atrophy and laparoscopy remains the solely way to exclude definitively the diagnosis (14). identify new prognostic factors for ovarian torsions. A comparison between THO and TUOM

134 in a pediatric population showed that: 1- THO is far more frequent than previously described 135 in children $(15,16)$. THO represents half of the cases in our series. 2- we were not able to 
differentiate THO and TUOM based on the clinical symptoms alone. 3- THO is associated with an increased risk of ovarian hypo/atrophy compared to TUOM.

We could have expected that an otherwise healthy ovary would have a better capacity of resistance to ischemia than an ovary with an additional pathological mass, but this is not the case. Two hypotheses may be proposed here. First, the diagnosis of THO may be more difficult and later than TUOM. The main sign for the diagnosis on ultrasonography is the increased size of the ovary. An underlying mass may facilitate the early visualization of an ovarian process. Diagnosis of an ovarian torsion was indeed more frequently suspected at the first visit in case of TUOM $(\mathrm{p}=0.005)$ and the time interval before surgery tended to be shorter in TUOM than in THO. But this did not reach significance and this may not be the solely explanation for the clear difference of prognosis between the two groups of patients.

Another hypothesis would be the role of the age of occurrence. Almost 9 out of 10 cases of THO hit prepubertal girls (vs 47.1\% TUOM, p=0.0004). From an endocrine point of view, prepubertal and postpubertal girls differentiate each other's by the plasma level of estrogens. This particular hormonal cellular environment may be relevant in case of ischemia. Recent studies showed that during the untwisting process, the revascularization of the ovary induces ischemia/reperfusion injuries through an oxidative stress pathway. Reactive species of oxygen (ROS), such as superoxide radical, hydrogen peroxide and hydroxyl radical are largely produced at this time and cause DNA damage and alteration of the cellular and mitochondrial membranes (17). Estrogens may modulate this oxidative stress that participates to ovarian hypotrophy. The ability of estradiol to reduce the level of ROS has been demonstrated both in in-vitro cell culture experiments and in numerous animal models as reviewed by Kumar et al. (18). Torsion of a healthy ovary during early infancy may thus be more deleterious than torsion with an underlying mass during puberty and estrogen impregnation.

Significant limitations remain in this study. Even if all patients were treated according to the same protocol (systematic ultrasonography, standardized follow-up, conservative management of the twisted ovary), this study remains retrospective and the absence of a systematic hormonal dosages after puberty (anti mullerian hormone) prevents to draw any definitive conclusions regarding the residual follicular reserve. 
To conclude, THO is more frequently associated with an ovarian atrophy or 171 hypotrophy than TUOM. A less obvious diagnosis at US and the early occurrence of THO 172 before puberty with a less favorable hormonal climate may explain this finding. One should 173 be particularly aware of THO in young girls even if clinical signs are not specific. 
Figure 1

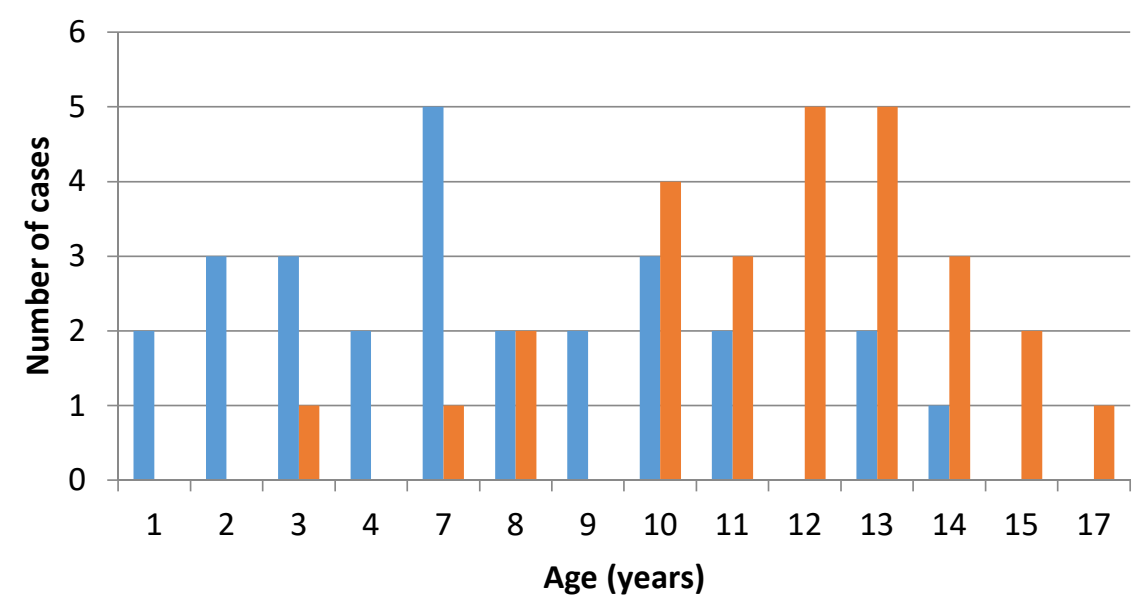

nealthy ovaries

pathological ovaries 
Figure 2

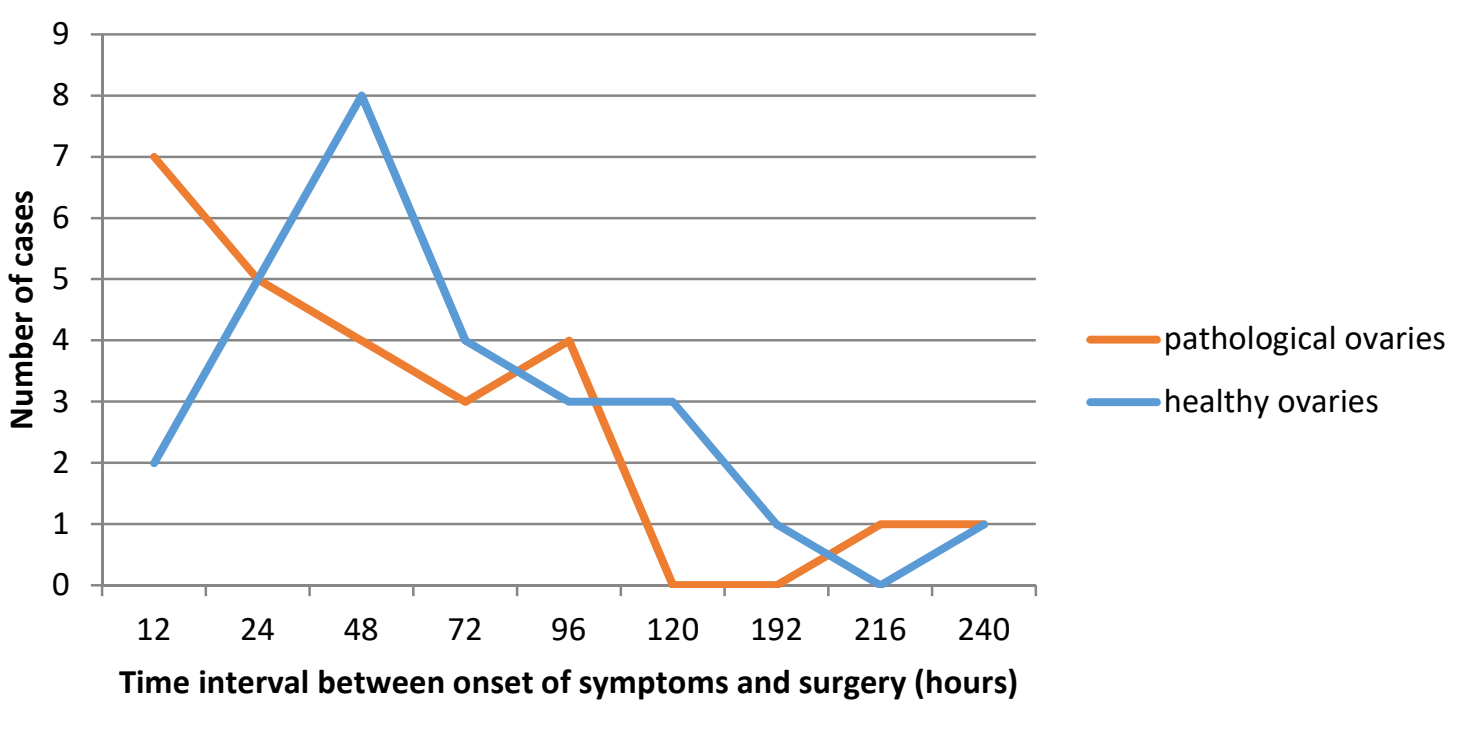


Table 1

\begin{tabular}{|c|c|c|c|}
\hline & $\begin{array}{l}\text { Healthy } \\
\mathrm{N}=27\end{array}$ & $\begin{array}{c}\text { Pathological } \\
\text { N=27 }\end{array}$ & $p$ value \\
\hline Age (mean) & $6.8(2-14)$ & $11.6(3-17)$ & $<.0001$ \\
\hline \multicolumn{4}{|l|}{ Abdominal pain } \\
\hline No & $0(0 \%)$ & $0(0 \%)$ & \multirow[t]{2}{*}{0.5860} \\
\hline Yes & $27(100 \%)$ & $27(100 \%)$ & \\
\hline \multicolumn{4}{|l|}{ Location } \\
\hline Pelvic median & $4(14.8 \%)$ & $10(37 \%)$ & \multirow[t]{4}{*}{0.1854} \\
\hline Right iliac fossa & $11(40.7 \%)$ & $5(18.5 \%)$ & \\
\hline Left iliac fossa & $6(22.2 \%)$ & $6(22.2 \%)$ & \\
\hline Diffuse & $6(22.2 \%)$ & $6(22.2 \%)$ & \\
\hline \multicolumn{4}{|l|}{ Vomiting } \\
\hline Yes & $10(37 \%)$ & $4(14.8 \%)$ & \multirow[t]{2}{*}{0.0624} \\
\hline No & $17(63 \%)$ & $23(85.2 \%)$ & \\
\hline \multicolumn{4}{|l|}{ Fever $\left(>38.5^{\circ} \mathrm{C}\right)$} \\
\hline Yes & $19(70.4 \%)$ & $23(85.2 \%)$ & \multirow[t]{2}{*}{0.1904} \\
\hline No & $8(29.6 \%)$ & $4(14.8 \%)$ & \\
\hline \multicolumn{4}{|l|}{ Abdominal tenderness } \\
\hline Yes & $4(14,8 \%)$ & $3(11.1 \%)$ & \\
\hline No & $23(85,2 \%)$ & $24(88,9 \%)$ & \\
\hline \multicolumn{4}{|c|}{ Initially suspected diagnosis } \\
\hline No precise diagnosis & $6(22.2 \%)$ & $0(0 \%)$ & \multirow[t]{6}{*}{0.0052} \\
\hline Torsion of the ovary & $8(29.6 \%)$ & $17(63 \%)$ & \\
\hline Acute intussusception & $4(14.8 \%)$ & $0(0 \%)$ & \\
\hline Acute appendicitis & $2(7.4 \%)$ & $2(7.4 \%)$ & \\
\hline $\begin{array}{l}\text { Hemorragic ovarian } \\
\text { cyst }\end{array}$ & $3(11.1 \%)$ & $7(25.9 \%)$ & \\
\hline Colitis / gastroenteritis & $4(14.8 \%)$ & $1(3.7 \%)$ & \\
\hline \begin{tabular}{|l} 
Time before surgery \\
$<12 \mathrm{~h}$
\end{tabular} & $7.4 \%(n=2)$ & $25.9 \%(\mathrm{n}=7)$ & 0.14 \\
\hline
\end{tabular}

